\title{
Conservative treatment of unruptured ectopic pregnancy in Jamaica
}

\author{
H. Fletcher, K. Buchanan, L. Jacob
}

Department of Obstetrics and Gynaecology, University of the West Indies, Mona, Kingston, Jamaica.

Email: horace.fletcher@uwimona.edu.jm

Received 15 February 2011; revised 18 March 2011; accepted 25 March 2011.

\begin{abstract}
Medical treatment with methotrexate is now successfully replacing conservative surgical treatment in selected patients. We reviewed patients treated with methotrexate, compared to salpingectomy and salpingostomy between 1990 to 1995 and 2000 to 2003. For 1990 - 1995 there were 21 conservative surgical treatments (5.3\% ectopic pregnancies). The ratio of ectopic to normal deliveries was 1:24. Medical treatment was done for 19 pregnancies (3.4\% of ectopic pregnancies) for 2000 to 2003. During the period there was an ectopic rate of 1:16.8 births. Patients treated medically were similar to those treated surgically except that none reportedly having rebound tenderness and only 1/18 having an ultrasound scan showing a foetal heart and none with a gestational sac $>\mathbf{4} \mathbf{c m}$. In the medically treated group the success rate was $68 \%$ while in the surgically treated groups success was $100 \%$. There was no significant difference in the parameters in the conservative surgical and medically treated groups.
\end{abstract}

Keywords: Conservative Treatment; Ectopic Pregnancy; Methotrexate; Linear Salpingostomy

\section{INTRODUCTION}

An ectopic pregnancy is the implantation of an embryo outside of the uterine cavity [1]. In the fallopian tube it will either be spontaneously reabsorbed, abort from the tube, or it may grow and rupture [2]. It is this latter type of ectopic pregnancy that is associated with maternal morbidity and mortality [3].

The maternal mortality rate in the United States has decreased to 3.4 per 10,000 cases of ectopic pregnancy with improvements in diagnosis and management [3]. In Jamaica during 3 years (1981 to 1983), ten percent of maternal deaths were from ectopic pregnancy [4].

Ectopic pregnancy may be treated medically or surgically. In the United States the method of treatment has changed from the primary surgical to medical treatment which now commonly predominates [5]. In Jamaica the primary mode of treatment still remains surgical as the patients tend to present later when signs and symptoms of rupture are more commonly present. At The University Hospital of The West Indies $96.8 \%$ and $92 \%$ of cases of ectopic pregnancies were treated surgically in 2002 and 2003 respectively. This hospital is also well known for an extremely high ectopic to delivery rate first reported by Douglas in 1963 of 1 ectopic in 29 pregnancies [6]. Surgical treatment is associated with the associated morbidity from both surgical and anaesthetic complications [7] and often results in the loss a fallopian tube which may have a bearing on the patient's fertility.

Surgery may also be done conservatively with linear salpingostomy salvaging the fallopian tube in cases where the ectopic is unruptured and the patient is stable. This method of treatment was more popular at UHWI in the 1990's before the advent of medical treatment of similar patients.

Medical management of ectopic pregnancy is done with the use of methotrexate. This is a folic acid antagonist that interferes with DNA synthesis and is effective against trophoblastic tissue. It was first used successfully in the treatment of ectopic pregnancy in Japan in 1982 [8]. The use of methotrexate decreases the need for surgical intervention and the associated morbidity. Methotrexate was also effective for ectopic pregnancies located in the cervix, ovary or cornua where surgical intervention carries significant risk [9]. However there is the associated risk of side effects due to the medication, the commonest of which are stomatitis and conjunctivitis but these side effects are usually mild and self-limiting. Thirty percent of patients who receive single dose management and $40 \%$ of those who receive multi-dose management experience side effects [10]. Also there is an associated failure rate $10 \%$ to $20 \%$ for the single dose therapy and $6 \%$ to $30 \%$ for the multidose therapy and this group of patients requires surgical intervention [1].

The success Medical management is dependent on the 
adherence to strict criteria. The success rate for single dose therapy has been reported to be $80 \%$ to $90 \%$ and $70 \%-94 \%$ for multi-dose therapy [1]. To employ medical treatment the patient must be:

1) Haemodynamically stable, reliable and compliant.

2) Ultrasonography should fail to show an intrauterine gestation and uterine curettage should fail to obtain villi

3) There should be no evidence of rupture of the ectopic pregnancy

4) The ectopic pregnancy should measure $4 \mathrm{~cm}$ or less in diameter

5) $\beta$ hCG titers should be $10000 \mathrm{IU} / \mathrm{L}$ or less

6) Relative contraindications include the presence of fetal cardiac activity on ultrasound.

The aim of this study was to look at conservative treatment of ectopic pregnancy assessing conservative surgery (linear salpingostomies) done 1990 - 1993 and also assessing the use of methotrexate management of ectopic pregnancy at this institution over the period 2000 to 2003 compared with standard surgery during the same period. The aim was to examine factors associated with failed versus successful treatment an also to see how strictly the above criteria was adhered to in patient selection for medical treatment treatment. We also examined the reproductive outcome of patients who received medical versus surgical treatment for the ectopic pregnancy and patient satisfaction of treatment.

\section{PATIENTS AND METHODS}

This was a retrospective observational study in which we compared medical therapy with methotrexate with radical surgical treatment (salpingectomy) during the period January 2000 to December 2003. The dockets of all the patients who presented with ectopic pregnancies from January 2000 to December 2003 were identified from the hospital database and we were able to find all the patients who had medical management of the ectopic pregnancy and these dockets were assessed for the study. We then randomly selected 19 dockets of patients from to use as controls using a system of random numbers given to the dockets of all patients who had surgical management of the ectopic pregnancy during the period January 2000 to December 2003.

We also reviewed the operations register in Main operating theatre for all cases of ectopic during the period June 1990 to July 1993 to identify all cases of conservative surgery (linear salpingostomy) for unruptured ectopic. We used this era, as this was a time when this operation was more popular. During this period we identified 21 patients who had this surgery but we were only able to locate 19 dockets (90.5\% case identification). These patients were also compared to the patients with methotrexate treatment.

In assessing reproductive outcome and patient satisfaction post treatment all patients in the medically treated and all patients in the surgically treated arm who were contactable by phone were given telephone interviews. They were asked if they had become pregnant since they received treatment and if that pregnancy was a repeat ectopic, or an intrauterine pregnancy and also if there was any history of secondary infertility after receiving treatment for their ectopic pregnancy. Also they were asked if they were satisfied with their treatment and outcome.

The odds ratio, P-values and confidence intervals (C.I) were calculated for each arm of the study for the presence of abdominal pain, per vaginal bleeding, both per vaginal bleeding and abdominal pain, nulliparity, abdominal tenderness, rebound tenderness, adnexal tenderness, gestational age less than 7 weeks, gestational age less than 10 weeks, the presence of shock, the presence of free fluid on ultrasound, gestational sac size greater than $4 \mathrm{~cm}$, the presence of cardiac activity on ultrasound, patient satisfaction, need for surgery, future pregnancy and repeat ectopic pregnancy after treatment.

\section{RESULTS}

Methotrexate treatment for ectopic pregnancy was done for 19 pregnancies in total for 2000 to 2003 . All 19 patients received a single dose regime. During the period there were 563 ectopic pregnancies and 9470 births (ectopic rate 1:16.8). Medical treatment was therefore done in $3.4 \%$ of ectopic pregnancies.

In all patients who were considered for methotrexate therapy a baseline quantitative serum $\beta$ hCG was done. Thirteen of these had abdominal pain and ten had abdominal tenderness but none had rebound tenderness. All of these patients had ultrasonography or diagnostic laparoscopy or a combination of both procedures prior medical therapy. Seventeen of these patients had ultrasonography, 5 had diagnostic laparoscopy and ultrasonography and one had diagnostic laparoscopy only. Seventeen patients had ultrasonography to rule out the presence of an intrauterine pregnancy. Of the 17 patients who had ultrasonography 12 had the gestational sac size commented on and all sac sizes that were less than $4 \mathrm{~cm}$. Those with failed therapy were more likely to have a sac

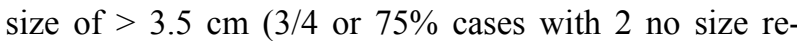
corded). While of those with successful treatment only $2 / 8$ or $25 \%$ had a sac size $>3.5 \mathrm{~cm}$. One patient had the presence of cardiac activity noted on ultrasound and she had failed medical treatment. The one patient who did not have ultrasonography, had her ectopic diagnosed at laparoscopy and the size was noted to be less than $4 \mathrm{~cm}$. Of the five patients who had diagnostic laparoscopy none 
had evidence of rupture of the ectopic gestation prior to commencing methotrexate.

Seventeen patients had at least one other quantitative serum $\beta \mathrm{hCG}$ done after the baseline $\beta \mathrm{hCG}$ was done, the eighteenth patient had only a baseline $\beta \mathrm{hCG}$ done but subsequently absconded from the ward. Six of the eighteen patients did not have follow up of the $\beta$ hCG as they had surgery due to failed medical management. Of the others only one patient had her $\beta$ hCG followed up until it was undetectable as outlined by the protocol. The pre-treatment range of $\beta \mathrm{hCG}$ for the medically treated group was 130 to $31860 \mathrm{mIU} / \mathrm{ml}$. Five of 19 patients and 10 of 19 patients in the medically treated group had $\beta$ hCG greater than $5000 \mathrm{mIU} / \mathrm{ml}$ and $3000 \mathrm{mIU} / \mathrm{ml}$ on day 1 respectively. Of the six patients who had failed therapy four $(66.7 \%)$ had $\beta \mathrm{hCG}>5000 \mathrm{mIU} / \mathrm{ml}$. While of the 13 who had successful medical therapy only 4 (30.7\%) had had $\beta \mathrm{hCG}>5000 \mathrm{mIU} / \mathrm{ml}$. However the patient with the highest level $\beta$ hCG (31 860) and longest gestational age 9 weeks had successful multiple dose treatment.

The duration of hospital stay commencing on the day that the Methotrexate therapy was started varied from 2 to 11 days. All patients had baseline complete blood counts done prior to Methotrexate therapy. Fourteen of the 18 patients had renal and liver function tests done and these were normal prior to starting Methotrexate. One patient had renal blood test done but no liver function test prior to commencing treatment (these results were normal) and 3 patients had no renal or liver function test done prior to the onset of Methotrexate treatment. Only 2 patients had a repeat of the renal and liver function tests having received the treatment and this was done appropriately on day 7 of Methotrexate treatment and these results were normal.

The dose of the methotrexate given could be verified as accurate in 10 of the eighteen patients as their weight and height were documented in their notes and hence the body surface area could be calculated. Three patients had only their weight documented and 5 patients had only their height documented. Of the ten patients who had their weight and height documented, 7 received an accurate Methotrexate dose, two received a dose that was higher than the correct recommended dose based on their body surface area (BSA) and one had a dose that was too low based on their BSA i.e. $50 \mathrm{mg}$ instead of $80 \mathrm{mg}$, however the medical treatment was still successful. Two patients received doses that were higher than that based on their BSA $100 \mathrm{mg}$ as against $71.5 \mathrm{mg}$ for one patient (however that patient's medical treatment was not successful and hence she needed surgery) and $100 \mathrm{mg}$ as against $75 \mathrm{mg}$ for the other patient (this patients medical therapy which was successful).
Five patients (26\%) had to have surgery (salpingectomy) because of failed treatment. Four patients, because of a rising $\beta \mathrm{hCG}$ for persistent ectopic on ultrasonography and one patient who went into shock after failed medical treatment.

Sixteen of the eighteen controls had salpingectomy at laparotomy. One patient had salpingostomy at laparotomy and one patient had laparoscopic salpingostomy. The median age of patients treated medically was 28 (range 21 - 36). The median age of patients treated surgically was 31 (range 22 - 42 years).

The overall success rate for methotrexate treated ectopic pregnancies was $68 \%$. The success rate for the surgically treated (salpingectomy) controls was $100 \%$.

The presence of adnexal tenderness and rebound tenderness were more common in the salpingectomy group. However there was no statistical significance difference noted between each group for abdominal pain, vaginal bleeding, nulliparity, abdominal tenderness, shock, gestational age below 7 weeks or above 10 weeks, ultrasound presence of cardiac activity, free fluid or gestational sac size more than $4 \mathrm{~cm}$. There was also no statistical significance noted between the two groups in terms of patient satisfaction, future pregnancy and future ectopic pregnancy (Table 1).

In the patients who had conservative surgery (salpingostomy) the clinical presentation findings were similar to those who had methotrexate treatment (Table 2). There were 9571 deliveries and 390 ectopic pregnancies during the era with $21(5.4 \%)$ conservative surgical treatment. The ratio of ectopic to normal deliveries was $1: 24$. The age range in this group was $20-38$ median value of 28 years old. A past history of ectopic was present in 5/19 patients and all had had a previous salpingectomy.

Fourteen patients had a positive pregnancy test however no quantitative $\beta$ hCG was offered during that period. Three patients had a culdo-centesis positive for non clotting blood. In fourteen cases an ultrasound scan was done showing and adnexal mass (size not recorded) and an empty uterus in 13 cases suggestive of ectopic pregnancy. Laparoscopy was done in six cases and in all cases the unruptured ectopic pregnancy was seen.

At surgery all patients had an unruptured ectopic pregnancy and linear salpingostomy was performed in all. Vasopressin was used for haemostasis in 7/19 cases and diathermy was used in $6 / 19$. The incision was closed in $10 / 19$ patients and was left open in 9/19. None of these patients had a failed procedure with repeat operation. Hospital stay ranged from 3 - 5 days with a median of 4 days. A summary of the comparison with the medically treated patients revealed no significant differences (Table 2). 
Table 1. A comparison of the clinical and laboratory findings in patients receiving medical (Methotrexate) vs surgical (salpingectomy) management of ectopic pregnancy.

\begin{tabular}{|c|c|c|c|c|}
\hline Variable & Medical & Surgical & Odd ratios $(\mathrm{CI})$ & P-values \\
\hline Nulliparous & $12 / 19(63.1 \%)$ & $5 / 18(27.8 \%)$ & $4.45(1.11-17.899)$ & 0.067 \\
\hline Abd pain & $13 / 19(68.4 \%)$ & $17 / 1(94.4 \%)$ & $0.127(0.014-1.193)$ & 0.11 \\
\hline Bleeding & $17 / 19(89.4 \%)$ & $13 / 18(72.2 \%)$ & $3.269(0.545-19.61)$ & 0.358 \\
\hline Abd pain bleeding & $13 / 19(68.4 \%)$ & $13 / 18(72.2 \%)$ & $0.833(0.203-3.427)$ & 0.915 \\
\hline Abdominal tenderness & $10 / 19(52.6 \%)$ & $15 / 18(83.3 \%)$ & $0.22(0.05-1.02)$ & 0.1 \\
\hline Rebound tenderness & $0 / 10(0 \%)$ & $11 / 18(61.1 \%)$ & $0.017(0.001-0.321)$ & 0.0001 \\
\hline Adnexal tenderness & $8 / 19(42.1 \%)$ & $16 / 18(88.9 \%)$ & $0.1(0.018-0.52)$ & 0.013 \\
\hline Free peritoneal fluid & $6 / 12(50 \%)$ & $10 / 14(71.4 \%)$ & $0.4(0.08-2.02)$ & 0.474 \\
\hline Shock & $1 / 19(5.2 \%)$ & $5 / 18(27.8 \%)$ & $0.063(0.003-1.24)$ & 0.065 \\
\hline Gest sac size $>4 \mathrm{~cm}$ & $2 / 12(16.7 \%)$ & 4/12 (33.3\%) & $0.25(0.034-1.863)$ & 0.365 \\
\hline Cardiac activity & $1 / 9(11.1 \%)$ & $4 / 8(50 \%)$ & $0.125(0.01-1.521)$ & 0.221 \\
\hline Surgery & $5 / 19(26.3 \%)$ & $18 / 18(100 \%)$ & $0.01(0.0010-0.201)$ & 0.0001 \\
\hline Gest age $<7$ weeks & $5 / 17(29.4 \%)$ & $4 / 17(23.5 \%)$ & $1.34(0.293-6.261)$ & 1.0 \\
\hline Gest age $>10$ weeks & $3 / 17(17.6 \%)$ & $0 / 17(0 \%)$ & $8.448(0.403-177.3)$ & 0.289 \\
\hline Repeat pregnancy & $2 / 7(28.5 \%)$ & $2 / 4(50 \%)$ & $0.4(0.031-5.151)$ & 0.953 \\
\hline Repeat ectopic pregnancy & $0 / 7(0 \%)$ & $1 / 4(25 \%)$ & $0.156(0.005-4.866)$ & 0.715 \\
\hline Satisfaction & $4 / 7(57.1 \%)$ & $4 / 4(100 \%)$ & $0.143(0.006-3.64)$ & 0.506 \\
\hline
\end{tabular}

Table 2. A comparison of the clinical and laboratory findings in patients receiving conservative medical (Methotrexate) vs conservative surgical (salpingostomy) management of ectopic pregnancy.

\begin{tabular}{lllll}
\hline Variable & Medical & Surgical & Odd ratios (CI) & P-values \\
\hline Nulliparous & $12 / 19(63.1 \%)$ & $11 / 19(58.0 \%)$ & $1.25(0.339-4.6)$ & 1.0 \\
Abd pain & $13 / 19(68.4 \%)$ & $15 / 19(79 \%)$ & $0.58(0.133-2.5)$ & 0.713 \\
Bleeding & $17 / 19(89.4 \%)$ & $15 / 19(79 \%)$ & $2.27(0.36-14.2)$ & 0.656 \\
Abd pain bleeding & $13 / 19(68.4 \%)$ & $9 / 19(47.4 \%)$ & $2.41(0.64-9.03)$ & 0.324 \\
Abdominal tenderness & $10 / 19(52.6 \%)$ & $13 / 16(68.4 \%)$ & $0.256(0.06-1.2)$ & 0.156 \\
Rebound tenderness & $0 / 10(0 \%)$ & $0 / 19(0 \%)$ & & 0.861 \\
Adnexal tenderness & $8 / 19(42.1 \%)$ & $2 / 7(28 \%)$ & $1.82(0.28-11.9)$ & 0.897 \\
Free peritoneal fluid & $6 / 12(50 \%)$ & $2 / 3(66.6 \%)$ & $0.5(0.035-7.10)$ & 1.0 \\
Shock & $1 / 19(5.2 \%)$ & $0 / 19(0 \%)$ & $3.2(0.12-82.6)$ & \\
Gest sac size $>4 \mathrm{~cm}$ & $2 / 12(16.7 \%)$ & Not available & & \\
Cardiac activity & $1 / 9(11.1 \%)$ & Not available & & 0.155 \\
Surgery & $5 / 19(26.3 \%)$ & $19 / 19(100 \%)$ & $0.36(0.11-1.19)$ & 0.825 \\
Gest age $<7$ weeks & $5 / 17(29.4 \%)$ & $6 / 19(31.6 \%)$ & $0.909(0.22-3.7)$ & 0.893 \\
Gest age $>10$ weeks & $3 / 17(17.6 \%)$ & $2 / 19(10.5 \%)$ & $1.82(0.27-12.5)$ & 0.242 \\
Repeat pregnancy & $2 / 7(28.5 \%)$ & $4 / 5(80 \%)$ & $0.1(0.006-1.54)$ & \\
Repeat ectopic pregnancy & $0 / 7(0 \%)$ & Not available & & \\
Satisfaction & $4 / 7(57.1 \%)$ & & &
\end{tabular}




\section{DISCUSSION}

The rate of ectopic pregnancies at the University of the West Indies Hospital, in this study is very high at 1:17 to 1:24 deliveries even higher than that reported in 1963 [6]. It is believed that this high rate is due to the fact that the hospital is a referral teaching hospital, with a low delivery rate for its size. The findings of this study revealed a lower success rate for single dose therapy than reported in the literature. The success rate for single dose methotrexate was $68 \%$ as against $80 \%-90 \%$ as quoted by Thoen et al [11]. The higher failure rate seen for medically managed patient in this study may have been that the criteria for medical management were not strictly adhered to as 13 of 19 patients and 10 of 19 patients medically treated had abdominal pain and abdominal tenderness (respectively) which may have been inherent signs of rupture. In one series the presence of the symptom of abdominal pain in the absence of abdominal tenderness resulted in a treatment failure $56 \%$ of the times as compared to $17 \%$ of the times when there was no abdominal pain or tenderness and the presence of vaginal bleeding was associated with a treatment failure $53 \%$ of the times compared with $16 \%$ when vaginal bleeding was present [12]. However Dilbaz et al [13] did not find that the presence of clinical signs and symptoms on admission predicted treatment failure.

Stika et al [14] found day $1 \beta \mathrm{hCG}$ levels $>5000$ $\mathrm{mIU} / \mathrm{ml}$ had a greater probability of requiring either surgical intervention or multidose methotrexate. Dilbaz et al [13] also found that a $\beta \mathrm{hCG}$ level above $3000 \mathrm{mIU} / \mathrm{ml}$ was predictive of treatment failure. It is essential to follow up the $\beta$ hCG levels until it is undetectable however only in one case in this series was the $\beta$ hCG followed up until it was undetectable. Lui et al [3] found that the initial $\beta \mathrm{hCG}$ level and the trend of the serial $\beta \mathrm{hCG}$ results were the most important factors that predicted the success of medical management. The risk of tubal rupture is $10 \%$ when the serum $\beta \mathrm{hCG}$ is less than 1000 $\mathrm{mIU} / \mathrm{ml}$ and rupture has been known to occur when the serum $\beta \mathrm{hCG}$ is less than $100 \mathrm{MIU} / \mathrm{ml}$ in isthmic pregnancies.

Cardiac activity has been associated with an increased risk of single dose methotrexate management failure [15] and the one patient medically treated who had presence of cardiac activity had failed management requiring surgery.

The presence of free fluid is not a contraindication for medical therapy with methotrexate [3]. The finding of free fluid on ultrasound is not an uncommon finding as trans-vaginal sonography can detect as little as $50 \mathrm{ml}$ of free fluid. The presence of free fluid is not diagnostic of a leaking ectopic pregnancy but should heighten the physician's awareness of the possibility of a leaking ec- topic pregnancy when the clinical context and other ultrasound findings are taken into consideration [16].

The presence of a gestational sac size greater than 5 $\mathrm{cm}$ is associated with an increased risk of treatment failure. Thoen et al found that a gestational sac size of more than $3.5 \mathrm{~cm}$ was associated with treatment failure [11]. Three of 13 patients had a gestational sac size of greater than $3.5 \mathrm{~cm}$ on ultrasound or laparoscopy but no patient had a sac size greater than $4 \mathrm{~cm}$.

The presence of rebound tenderness is a sign of irritation of the peritoneal lining, in the case of a patient presenting with an ectopic pregnancy this is usually due to blood from the site of rupture of the ectopic pregnancy irritating the peritoneal lining [17]. Patients who were treated with salpingectomy were more likely to have adnexal tenderness and this was statistically significant ( $p$ value $<0.013$ ).

The future pregnancy rate was $50 \%$ for salpingectomy and $28.5 \%$ for methotrexate treated ectopic pregnancy in our study. Bouyer et al [17] found the intrauterine pregnancy rates to be lower for salpingectomy treatment and higher for medical treatment. He also found the intrauterine pregnancy rates to be lower for conservative surgical treatment than for medical treatment and higher for conservative surgical than radical surgical treatment [18]. The findings in our study may be out of keeping with others because of poor follow-up of all the patients.

The rate of repeat ectopic pregnancy was $0 \%$ in the medically treated group and $25 \%$ in the salpingectomy treated group. Bouyer et al [17] found that the rate of recurrent ectopic was not different based on the treatment but found that other factors including presence of tubular disease affected reproductive outcome and the risk of ectopic pregnancy [18].

In this study only 14 of the 18 patients had both baseline renal and liver function tests done. Serious and potentially fatal side effects can occur in the presence of liver or renal failure with methotrexate.

This study was limited by the presence of a small sample size and this may have limited the number of results that were significant. Another limiting factor was that this was a retrospective study and hence is more prone to error as against a prospective study due to confounding factors and bias. There was difficulty and in many cases it was not possible to contact many patients.

This small study however confirms that medical therapy is a viable option in carefully selected patients however we still have to overcome challenges of follow-up of treatment in our setting.

\section{REFERENCES}

[1] Centers for Disease Control and Prevention (1995) Ec- 
topic pregnancy - United States 1990-1992. Morbidity and Mortality Weekly Report, 44, 46-48.

[2] Kamwendo, F., Forslin, L., Bodin, L. and Danielsson, D. (2000) Epidemilogy of ectopic pregnancy during a 28 year period and the role of pelvic inflammatory disease. Sex Transmitted Infect, 76, 28-32. doi:10.1136/sti.76.1.28

[3] Lui, A., D’Ottavio, G., Rustico, M.A., Conoscenti, G., Fischer Tamaro, F., Meir, Y.J., Maieron, A. and Mandruzzato, G.P. (1997) Conservative management of ectopic pregnancy. Minerva Ginecologica, 49, 67-72.

[4] Walker, G.J.A., Ashley, D.E.C., McCaw, A. and Bernard, G.W. (1986) Maternal mortality in Jamaica. Lancet, 486-488. doi:10.1016/S0140-6736(86)92939-9

[5] Yao, M. and Tulandi, T. (1997) Current status of surgical and non-surgical management of ectopic pregnancy. Fertility and Sterility, 67, 421-433. doi:10.1016/S0015-0282(97)80064-7

[6] Douglas, C.P. (1963) Tubal ectopic pregnancy. British Medical Journal, 2, 838. doi:10.1136/bmj.2.5361.838

[7] Landström, G., Thorburn, J. and Bryman, I. (1998) Treatment, failures and complications of ectopic pregnancy: Changes over a 20 year period. Human Reproduction, 13, 203-207.

[8] Tanaka, T., Hayashi, H., Kutsuzawa, T. and Ichinoe, K. (1982) Treatment of interstitial ectopic pregnancy with methotrexate: Report of a successful case. Fertility and Sterility, 37, 851-852.

[9] Yankowitz, J., Leake, J., Huggins, G., Gazaway, P. and Gates, E. (1990) Cervical ectopic pregnancy: A review of the literature and report of a case treated by single dose methotrexate therapy. Obstetrical \& Gynecological Survey, 45, 405-414. doi:10.1097/00006254-199007000-00001

[10] Barnhart, K., Gosman, M., Ashby, R. and Sammel, M. (2003) The medical management of ectopic pregnancy: A meta-analysis comparing "single-dose" and "multi-dose" regimens. Obstetrics \& Gynecology, 101, 778-784.
doi:10.1016/S0029-7844(02)03158-7

[11] Thoen, L. and Crenin, M.D. (1997) Medical treatment of ectopic pregnancy with methotrexate. Fertility and Sterility, 68, 727-730. doi:10.1016/S0015-0282(97)00284-7

[12] Tawfig, A., Agameya, A.F. and Claman, P. (2000) Predictors of treatment failure for ectopic pregnancy treated with single dose methotrexate. Fertility and Sterility, 74, 877-880. doi:10.1016/S0015-0282(00)01547-8

[13] Dilbaz, S., Caliskan, E., Dilbaz, B., Degirmenci, O. and Haberal, A. (2006) Predictors of methotrexate treatment failure in ectopic pregnancy. Journal of Reproductive Medicine, 51, 87-93.

[14] Stika, C.S., Anderson, L. and Frederiksen, M.C. (1996) Single dose methotrexate for the treatment of ectopic pregnancy. North Western Memorial Hospital 3 year experience. American Journal of Obstetrics \& Gynec- ology, 174, 1840-1846. doi:10.1016/S0002-9378(96)70219-3

[15] Erdem, A., Arslam, H., Biberoglu, K. and Gursoy, R. (2004) Single dose methotrexate for the treatment of unruptured ectopic pregnancy. Archives of Gynecology \& Obstetrics, 270, 201-204. doi:10.1007/s00404-003-0543-4

[16] Condous, G., Okaro, E., Khalid, A., Lu, C. and Van Huffel, S. (2005) The accuracy of transvaginal ultrasonography for the diagnosis of ectopic pregnancy prior to surgery, Human Reproduction, 20, 1404-1409. doi:10.1093/humrep/deh770

[17] Bouyer, J., Job-Spira, N., Pouly, J.L., Coste, J., Germaine, E. and Fernandez, H. (2000) Fertility following radical, conservative-surgical or medical treatment for tubal pregnancy: A population based study. British Journal of $\mathrm{Ob}$ stetrics \& Gynecolog, 107, 714-721.

[18] Kelly, H. and Harvey, D. (2006) A cautionary tale: A fatal outcome of methotrexate therapy given for management of ectopic pregnancy. Obstetrics \& Gynecology, 107, 439-441. doi:10.1097/01.AOG.0000172374.72125.3e 